\title{
Correction to: Picomolar concentrations of oligomeric alpha-synuclein sensitizes TLR4 to play an initiating role in Parkinson's disease pathogenesis
}

\author{
Craig D. Hughes ${ }^{1,2} \cdot$ Minee L. Choi ${ }^{3,4} \cdot$ Mina Ryten $^{5} \cdot$ Lee Hopkins $^{1} \cdot$ Anna Drews ${ }^{2,9} \cdot$ Juan A. Botía $a^{5,6} \cdot$ Maria Iljina $^{2,10}$. \\ Margarida Rodrigues $^{2} \cdot$ Sarah A. Gagliano ${ }^{7} \cdot$ Sonia Gandhi ${ }^{4,5} \cdot$ Clare Bryant $^{1} \cdot$ David Klenerman $^{2,8}$ (I)
}

Published online: 17 October 2018

(c) Springer-Verlag GmbH Germany, part of Springer Nature 2018

\section{Correction to: Acta Neuropathologica https://doi.org/10.1007/s00401-018-1907-y}

In the original publication of this article, the author Magarida Rodrigues was written incorrectly. The correct spelling should have been Margarida Rodrigues. Also the centrifuge speed used to remove fibrillar pellets from the aggregation reaction was written incorrectly as $14.2 \mathrm{rpm}$ and should have been $14,200 \mathrm{rpm}$. The original article has now been updated to reflect these changes.

The original article can be found online at https://doi.org/10.1007/ s00401-018-1907-y.

Sonia Gandhi

sonia.gandhi@ucl.ac.uk

$\triangle$ Clare Bryant

ceb27@cam.ac.uk

$\triangle$ David Klenerman dk10012@cam.ac.uk

1 Department of Veterinary Medicine, University of Cambridge, Cambridge CB3 OES, UK

2 Department of Chemistry, University of Cambridge, Cambridge CB2 1EW, UK

3 Department of Clinical and Movement Neuroscience, UCL Institute of Neurology, Queen Square, London WC1N 3BG, UK

4 The Francis Crick Institute, 1 Midland Road, London NW1 1AT, UK
5 Department of Molecular Neuroscience, University College London Institute of Neurology, London WC1N 3BG, UK

6 Departamento de Ingeniería de la Información y las Comunicaciones, Universidad de Murcia, 30100 Murcia, Spain

7 Center for Statistical Genetics, University of Michigan, Ann Arbor, MI, USA

8 UK Dementia Research Institute, University of Cambridge, Cambridge CB2 0XY, UK

9 Present Address: German Center for Neurodegenerative Diseases, Sigmund-Freud-Straße 27, 53127 Bonn, Germany

10 Present Address: Weizmann Institute of Science, Perlman Chemical Sciences Building, Room 601, 76100 Rehovot, Israel 